\title{
Comparison Study on Changes of Antigenicities of Egg Ovalbumin Irradiated by Electron Beam or X-Ray
}

\author{
Mi-Jung Kim¹, Ju-Woon Lee, Nak-Yoon Sung, Su-Min Kim, Young-Jung Hwang², \\ Jae-Hun Kim, and Beom-Seok Song* \\ Advanced Radiation Technology Institute, Korea Atomic Energy Research Institute, Jeongeup 580-185, Korea \\ ${ }^{1}$ Department of Food Science and Nutrition, Anyang University, Anyang 430-714, Korea \\ ${ }^{2}$ Department of Food Service and Culinary, International University of Korea, Jinju 660-759, Korea
}

\begin{abstract}
This study was conducted to compare the effects of two forms of radiation (electron and X-ray; generated by an electron beam accelerator) on the conformation and antigenic properties of hen's egg albumin, ovalbumin (OVA), which was used as a model protein. OVA solutions $(2.0 \mathrm{mg} / \mathrm{mL})$ were individually irradiated by electron beam or X-ray at the absorbed doses of 0 (control), 2, 4, 6, 8, and $10 \mathrm{kGy}$. No differences between the two forms of radiation on the structural properties of OVA were shown by spectrometric and electrophoretic analyses. The turbidity of OVA solution increased and the main OVA bands on polyacrylamide gels disappeared after irradiation, regardless of the radiation source. In competitive indirect enzyme-linked immunosorbent assay, OVA samples irradiated by electron beam or X-ray showed different immunological responses in reactions with monoclonal and polyclonal antibodies (immunoglobulin G) produced against non-irradiated OVA. The results indicate that electron beam irradiation and X-ray irradiation produced different patterns of structural changes to the OVA molecule.
\end{abstract}

Keywords: electron beam, X-ray, ovalbumin, conformational change

\section{Introduction}

The use of electron beams and X-rays generated by a particle accelerator (a machine source of radiation) has slightly increased in food irradiation and sterilization technology (Miller, 2003). A large proportion of industrial gamma irradiation may be replaced by the use of powerful electron accelerators, because of the lack of public acceptance and the inconvenience involved in the international trade in radioactive materials such as Cobalt-60 or Cecium-137, which are used to generate gamma rays (Cleland and Stichelbaut, 2013). It is expected that X-ray radiation will be used more widely in food and sterilization industries due to its equivalent effectiveness on final products compared with gamma rays (Palou et al., 2007; van Calenberg et al., 1998, 1999). However, the low efficacy of the energy of X-ray irradiation should be improved for more practical and wider applications in

*Corresponding author: Beom-Seok Song, Advanced Radiation Technology Institute, Korea Atomic Energy Research Institute, Jeongeup 580-185, Korea. Tel: +82-63-570-3211, Fax: 82-63570-3207, E-mail: sbs0110@kaeri.re.kr industry (Byun et al., 2009).

The effects of ionizing energy on food compounds have been studied over wide ranges for various foods. Effects among the three radiation sources (gamma ray, electron beam, and X-ray) are not obviously different at the macroscopic level. However, at the microscopic level, food compounds are exposed directly to electrons during electron beam irradiation and this may induce different effects on food compounds, compared with gamma and X-ray irradiation (Thayer, 1984; van Calenberg et al., 1999). Therefore, a comparison study should be performed to understand the properties of reactions at the molecular level between food compounds and different sources of ionizing radiation.

In this study, isolated hen's egg albumin (ovalbumin, OVA) was used as a model protein to examine the differences between electron beam irradiation and X-ray irradiation in terms of conformational changes to OVA. OVA was selected because the structural changes caused by gamma irradiation have been relatively well explained in several studies (Kim et al., 2002; Kume et al., 1994; Lee at al., 2001), and these reports can be used as references in this comparison study between electron beam and X- 
ray irradiations. Turbidity at ultraviolet (UV) wavelengths, electrophoretic patterns on a sodium dodecyl polyacrylamide gel (SDS-PAGE), and immunological responses between antibody $(\mathrm{Ab})$ and antigen $(\mathrm{Ag})$ in competitive indirect enzyme-linked immunosorbent assay (Ci-ELISA) were measured to evaluate the differences in conformational changes to OVA induced by ionizing radiation that was generated by two radiation sources.

\section{Materials and Methods}

\section{Protein and antibodies}

OVA (purified from egg white) was purchased from Sigma Chemical Co. (USA) for use as the standard antigen. Mouse monoclonal $\mathrm{Ab}(\mathrm{M}-\mathrm{IgG})$ and polyclonal $\mathrm{Ab}$ (P-IgG) for the testing of binding activity to OVA were purchased from LifeSpan Biosciences (USA). Horseradish peroxidase- (HRP-) conjugated rabbit anti-mouse IgG (Santa Cruz Biotechnology, USA) was used as a secondary $\mathrm{Ab}$ for $\mathrm{M}-\mathrm{IgG}$ - and $\mathrm{P}-\mathrm{IgG}$-formatted Ci-ELISAs.

\section{Preparation of OVA solution}

OVA was dissolved in $0.01 \mathrm{M}$ sodium phosphate-buffered saline (PBS) containing $0.15 \mathrm{M} \mathrm{NaCl}(\mathrm{pH} 7.4)$ and adjusted to a final concentration of $2.0 \mathrm{mg} / \mathrm{mL}$ by comparing it with a standard protein solution prepared commercially. The concentrations of OVA solutions were determined by the bicinchoninic acid (BCA) method (Hill and Straka, 1988) using a BCA protein assay kit (Sigma Chemical Co.). Bovine serum albumin (BSA; Sigma Chemical Co.) solution $(0.5-4.0 \mathrm{mg} / \mathrm{mL})$ was used as a standard.

\section{Electron beam and $X$-ray irradiations}

OVA solution $(5 \mathrm{~mL})$ was put into glass tubes $(1 \mathrm{~cm}$ diameter). The tubes were placed horizontally onto plastic trays, tied with adhesive tape, and irradiated at the designated doses of 0 (control), 2, 4, 6, 8 and $10 \mathrm{kGy}$. Radiochromic film dosimetry with an area of $2 \mathrm{~cm}^{2}$ (Gafchormic $^{\circledR}$ HD-810, International Specialty Products, USA) was placed at the top and bottom of the tube (height of $1 \mathrm{~cm}$ ) for each sample. Electron beam irradiation and X-ray irradiation were applied using an ELV-4 Electron-BeamAccelerator (2.5 MeV) and an X-Ray Linear Accelerator (15 Kw, $5 \mathrm{MeV}$ ), respectively, at EB-Tech (EB-Tech Co., Korea). Optical densities (OD) of the dosimetry films were measured using a spectrophotometer at $560 \mathrm{~nm}$ and the actual absorbed doses were calculated by a method reported previously (van Clenberg et al., 1999). After irra- diation, the sample tubes were stored at $4^{\circ} \mathrm{C}$ and used in subsequent experiments.

\section{Determination of turbidity}

Turbidity was measured with a spectrophotometer using a modification to the method of Chan et al. (1993) and Lee et al. (2000b). Irradiated OVA solutions $(2.0 \mathrm{mg} / \mathrm{mL})$ were placed in a quartz cuvette (1 cm light path length) and OD values were measured at 280, 320, 340, and 660 $\mathrm{nm}$.

\section{Gradient sodium dodecyl sulfate-polyacrylamide (SDS-PAGE)}

Samples of irradiated OVA solutions $(2.0 \mathrm{mg} / \mathrm{mL})$ were resolved by gradient SDS-PAGE (4-12\% polyacrylamide gel) performed using Laemmli's method (1970). Staining and destaining of gels was performed using the method of Lee et al. (2000b). Destained gels were scanned using an image analysis system (Molecular Analyst with windows software for Bio-Rad, Version 1.5, 1992-1995, Bio Rad Pacific Ltd, USA) and OD values were presented as relative intensity. The ratio of the OD value of the irradiated OVA band to the OD of the non-irradiated OVA band was calculated to represent the relative band intensity percentage (\%). A SeeBlue Plus2 Pre-stained standard marker was purchased from Invitrogen (USA) to determine the molecular masses of the protein bands. The standard protein marker contains 260, 160, 110, 80, 60, 50, 40, 30, 20, 15,10 , and $3.5 \mathrm{kDa}$ standards.

\section{Ci-ELISA}

Ci-ELISAs were individually formatted with M-IgG and P-IgG using previously reported methods (Kim et al., 2002; Lee et al., 2001) with slight modifications. Briefly, polystyrene flat-bottom microtiter plates (Maxisorp, Nunc, Denmark) were coated with $100 \mu \mathrm{L}$ of non-irradiated OVA dissolved $(10 \mu \mathrm{g} / \mathrm{mL})$ in a $0.2 \mathrm{M}$ bicarbonate buffer ( $\mathrm{pH}$ 9.6), and incubated overnight at $4^{\circ} \mathrm{C}$. The plates were washed three times with PBS containing $0.01 \%(\mathrm{v} / \mathrm{v})$ Tween 20 (PBST). To reduce non-specific binding, the plates were blocked with $200 \mu \mathrm{L}$ of PBS containing $2 \%$ (w/v) BSA. After washing, $50 \mu \mathrm{L}$ samples of various concentrations $(1.5,3.1,6.2,12.5,25.0,50.0$, and $100.0 \mu \mathrm{g} /$ $\mathrm{mL})$ of the non-irradiated and irradiated $(2,4,6,8$, and 10 kGy) OVA solutions were added to each well, and then $50 \mu \mathrm{L}$ of $\mathrm{M}$-IgG or P-IgG (LifeSpan Biosciences, USA) diluted in PBS (M-IgG: 640,000-fold, P-IgG: 80,000fold) was added. The plates were incubated for $2 \mathrm{~h}$, and then washed three times with PBST. To detect OVA bind- 
ing to $\mathrm{M}-\mathrm{IgG}$ or P-IgG on the well, $100 \mu \mathrm{L}$ of HRP-conjugated goat anti-mouse IgG antibody (Santa Cruz Biotechnology) solution diluted 1:2,000 in PBS was added to each well and incubated for $1 \mathrm{~h}$. The plates were then washed, and $100 \mu \mathrm{L}$ of 3,3,5,5-tetramethylbenzidine (eBioscience, USA) substrate solution was added for $15 \mathrm{~min}$ to produce a color reaction, before stopping the reaction with $50 \mu \mathrm{L}$ of $0.1 \mathrm{M} \mathrm{H}_{2} \mathrm{SO}_{4}$. The absorbance was measured at $450 \mathrm{~nm}$ using an ELISA plate reader (Varioskan Flash 3001, Thermo Scientific, USA).

\section{Statistical analysis}

All samples were tested in triplicate, and the experiments were repeated five times. Means and standard deviations calculated from the equations for $\mathrm{M}-\operatorname{IgG}$ and $\mathrm{P}-$ IgG were used to evaluate the binding capacity (detected concentration) of OVA. Data obtained were analyzed by general linear procedures, least square means with limited standard deviations, and Duncan's multiple-range test as programmed by SAS ${ }^{\circledR}$ software (SAS Institute, 2008).

\section{Results and Discussion}

\section{The changes of turbidity of OVA solutions}

Irradiated OVA solution $(2.0 \mathrm{mg} / \mathrm{mL})$ became increasingly turbid at increasing doses, regardless of radiation sources (Table 1). The increase in turbidity of irradiated OVA solution caused an increase in OD values at a low UV wavelength ( $\leq 360 \mathrm{~nm}$ ). Exposure of hydrophobic residues of inner parts of the protein by a low radiation dose might increase the turbidity of the OVA solution (FilaliMouthim et al., 1997; Lee and Byun, 2003). The structural release of susceptible parts in OVA might progress with irradiation at low doses of 2-4 kGy. At higher doses of $6 \mathrm{kGy}$ and above, OVA might start to break down into polypeptides or become agglutinated, and then the native OVA could change into other molecules with new molecular weights and forms (Al-kahtani et al., 1998; Kume et al., 1994). This result is very similar to that reported in a previous study (Lee et al., 2001), which indicated that gamma-irradiated OVA solutions became increasingly turbid depending on the dose. Lee et al. (2000b) also reported an increase in turbidity of bovine myosin solution with gamma irradiation. Meanwhile, an increase in OD values at $280 \mathrm{~nm}$ can be misinterpreted as an increase in concentration of the protein. In the BCA assay, colorimetric changes were not observed in all samples. The result did not indicate changes in concentration but changes to the structure by ionizing radiation (Byun et al., 2000).

\section{The changes in electrophoretic patterns}

On SDS-PAGE, intact OVA showed two bands, a 43$\mathrm{kDa}$ major band and its dimer at approximately $80 \mathrm{kDa}$ (Fig. 1). The change to OVA bands caused by electron beam or X-ray irradiation could be visually observed from $2 \mathrm{kGy}$ in both irradiated samples. A band at $80 \mathrm{kDa}$ and the major band $(43 \mathrm{kDa}$ ) were not found in $2 \mathrm{kGy}$ irradiated samples and $4 \mathrm{kGy}$-irradiated samples, respectively. New bands were not observed, and traces of samples were smeared from the top of separating gels at doses of $4 \mathrm{kGy}$ or more. However, the generation of new bands and traces was not shown in the lower molecular weight zone $(<80 \mathrm{kDa})$. Any visible differences between electron beam irradiation and X-ray irradiation were not evident on the gels. The relative band intensity (43 kDa) significantly decreased dependent upon the dose. The band intensities of OVA irradiated at $10 \mathrm{kGy}$ by both forms of radiation decreased more than $90 \%$, compared

Table 1. Changes in turbidity and colorimetric reaction by bicinchoninic acid assay of ovalbumin solution $(2.0 \mathrm{mg} / \mathrm{mL}) \mathrm{irradi}-$ ated by electron beam or X-ray

\begin{tabular}{|c|c|c|c|c|c|c|c|}
\hline \multirow{2}{*}{$\begin{array}{c}\text { Wavelength } \\
\text { (nm) }\end{array}$} & \multirow{2}{*}{ Radiation source } & \multicolumn{6}{|c|}{ Irradiation dose (kGy) } \\
\hline & & 0 & 2 & 4 & 6 & 8 & 10 \\
\hline \multirow{2}{*}{280} & Electron beam & $1.09^{a}$ & $1.52^{b}$ & $1.81^{c}$ & $1.98^{d}$ & $2.06^{e}$ & $2.10^{e}$ \\
\hline & X-ray & $1.07^{a}$ & $1.51^{b}$ & $1.82^{c}$ & $1.93^{d}$ & $2.02^{e}$ & $2.09^{f}$ \\
\hline \multirow{2}{*}{320} & Electron beam & $0.12^{a}$ & $0.26^{b}$ & $0.39^{c}$ & $0.51^{d}$ & $0.59^{d e}$ & $0.67^{e}$ \\
\hline & X-ray & $0.10^{a}$ & $0.26^{b}$ & $0.38^{c}$ & $0.49^{d}$ & $0.57^{e}$ & $0.68^{f}$ \\
\hline \multirow{2}{*}{340} & Electron beam & $0.10^{a}$ & $0.19^{b}$ & $0.27^{b c}$ & $0.35^{c}$ & $0.39^{c d}$ & $0.45^{d}$ \\
\hline & X-ray & $0.09^{a}$ & $0.19^{b}$ & $0.26^{b c}$ & $0.33^{c}$ & $0.38^{c d}$ & $0.45^{d}$ \\
\hline \multirow{2}{*}{660} & Electron beam & 0.07 & 0.07 & 0.06 & 0.07 & 0.07 & 0.07 \\
\hline & X-ray & 0.06 & 0.07 & 0.06 & 0.06 & 0.07 & 0.07 \\
\hline \multirow{2}{*}{$562^{1)}$} & Electron beam & 0.39 & 0.38 & 0.39 & 0.39 & 0.40 & 0.39 \\
\hline & X-ray & 0.39 & 0.39 & 0.39 & 0.38 & 0.39 & 0.39 \\
\hline
\end{tabular}

$\overline{a-f}$ Different letters within a row with the same wavelength indicate significant differences $(p<0.05)$.

${ }^{1)}$ OD values of OVA solutions were measured by bicinchoninic acid assay based on the colorimetric reaction. 

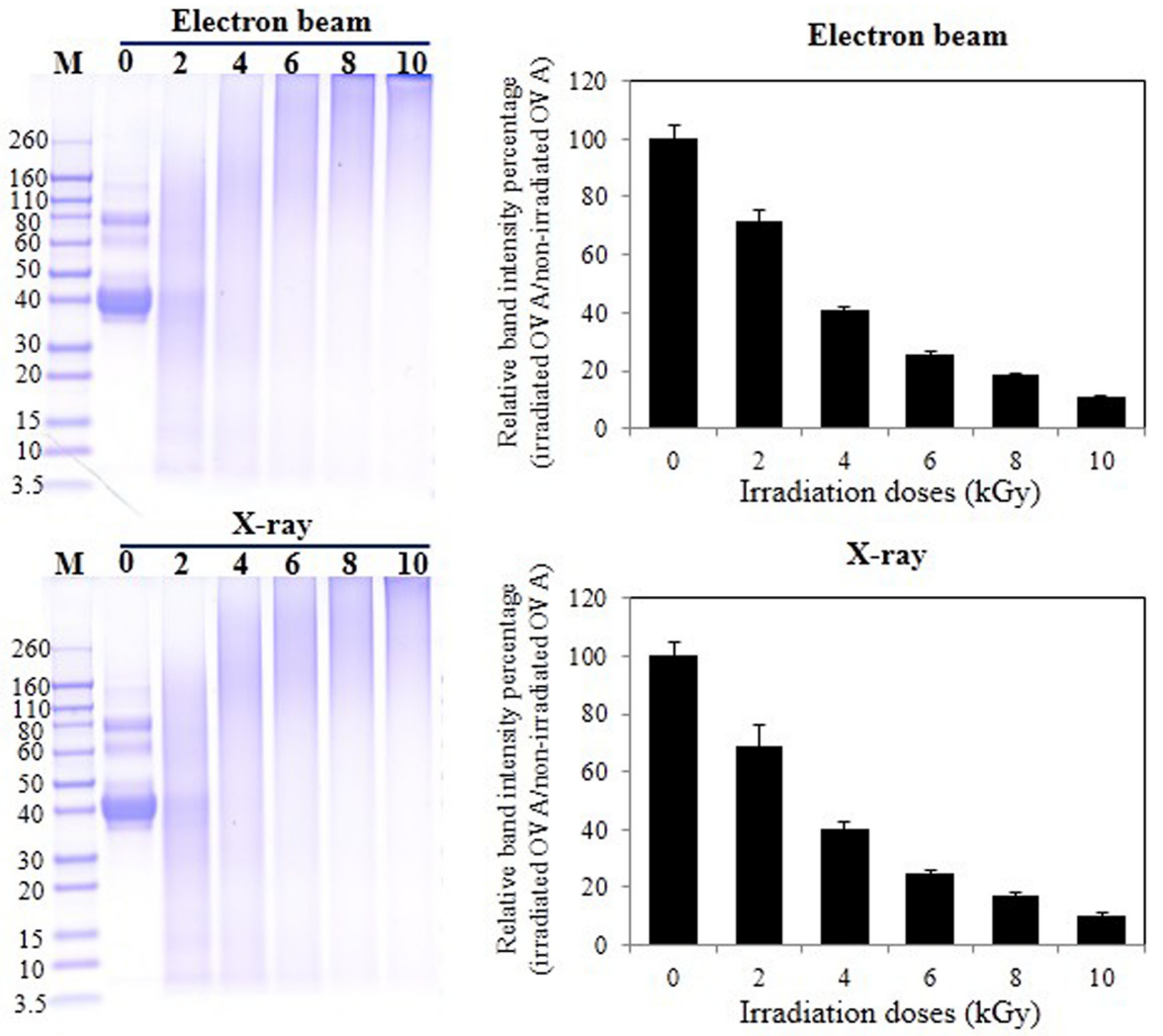

Fig. 1. Electrophoretic patterns [(A), gradient SDS-PAGE, 4-12\% polyacrylamide gel] and densitometric values [(B), relative band intensity (\%), irradiated OVA/non-irradiated OVA] of OVA on gels. M, prestained molecular weight marker; $0,0 \mathrm{kGy}$; 2, 2 kGy; 4, 4 kGy; 6, 6 kGy; 8, 8 kGy; 10, 10 kGy.

with the non-irradiated OVA band (Fig. 1B). Several researchers have reported similar results for gamma-irradiated protein solutions, such as shrimp tropomyosin (Byun et al., 2000), milk proteins (Cho et al., 2001; Lee et al., 2000a), bovine skeletal-muscle myosin (Lee et al., 2000b), and egg proteins (Kang et al., 2002; Kim et al., 2002; Kume and Matsuda, 1995). However, this result appeared to be opposite to the result of Filali-Mouhim et al. (1997). By ionizing radiation, a protein molecule can be broken down to smaller molecules or aggregate to form larger molecules by interactions among the individual molecules (Kume and Matsuda, 1995; Kuntz and White, 1961). OVA became coagulated and formed very large aggregates after electron beam or X-ray irradiation in the presence of oxygen and at neutral $\mathrm{pH}$ condition ( $\mathrm{pH}$ 7.4).

The changes in M-IgG- and P-IgG-binding capacities of OVA irradiated by electron beam or $X$-ray

Changes in the binding capacity (detected concentration, $\mu \mathrm{g} / \mathrm{mL}$ ) of OVA for M-IgG or P-IgG in OVA solutions irradiated by electron beam or X-ray were observed by Ci-ELISA. Fig. 2 indicates the binding capacities of
M-IgG against each OVA sample irradiated by electron beam or X-ray. The binding capacity of M-IgG increased towards 4-kGy electron beam-irradiated OVA (about 6.45 times), then decreased at higher doses. In X-ray-irradiated OVA samples, $\mathrm{M}-\mathrm{IgG}$ recognized OVA irradiated at $2 \mathrm{kGy}$ and above better than non-irradiated samples, and the binding capacity was strongest at $4 \mathrm{kGy}$ with a reactivity of about 5.9 times. The binding capacity of M-IgG to Xray-irradiated OVA decreased at doses higher than $6 \mathrm{kGy}$. However, differences were not statistically significant between $4 \mathrm{kGy}$ and $8 \mathrm{kGy}$ in electron beam-irradiated OVA and between $2 \mathrm{kGy}$ and $6 \mathrm{kGy}$ in X-ray-irradiated OVA $(p>0.05)$. M-IgG reacted with electron beam-irradiated OVA better than with X-ray-irradiated OVA. Statistically significant differences were observed between radiation sources for the binding capacity of $\mathrm{M}-\operatorname{IgG}(p<0.05)$.

Fig. 3 indicates the binding capacities of P-IgG against each OVA sample irradiated by electron beam or X-ray. The binding capacity of P-IgG was higher for irradiated OVA samples than non-irradiated samples, regardless of radiation source. Binding capacity increased for 6-kGy electron beam-irradiated OVA with a reactivity of about 


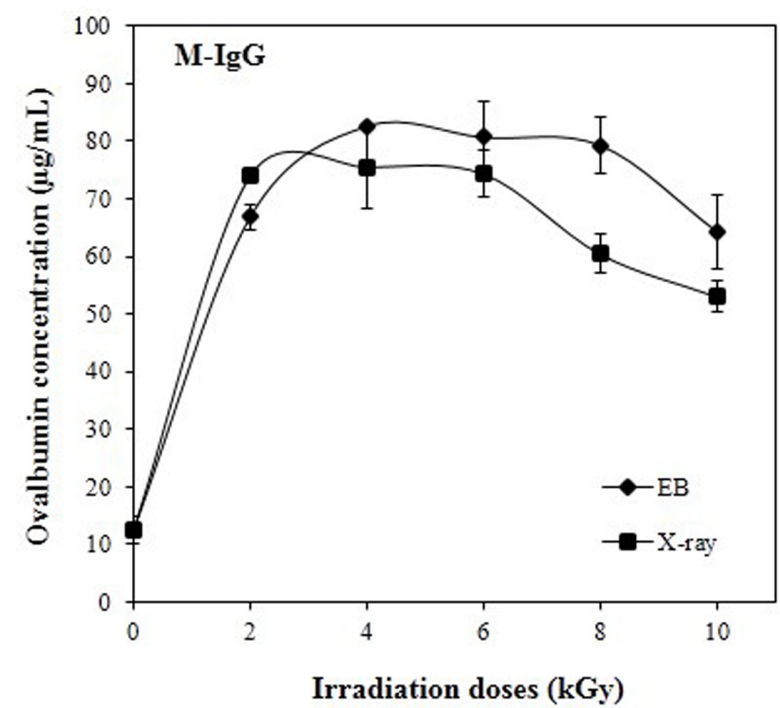

Fig. 2. Mouse murine monoclonal IgG-binding capacities (detected concentration, $\mu \mathrm{g} / \mathrm{mL}$ ) of each OVA sample irradiated by electron beam or X-ray. The OVA concentrations were individually calculated using equations derived from standard curves obtained by Ci-ELISA formatted with the mouse murine monoclonal IgG against electron beam-irradiated OVA (Electron beam, $\bullet$ ) or X-ray-irradiated OVA (X-ray, $\boldsymbol{\square})$.

8.4 times, and then decreased at higher doses. In X-rayirradiated OVA samples, P-IgG recognized OVA irradiated at $2 \mathrm{kGy}$ and above better than non-irradiated samples, and the binding capacity increased in a dose-dependent manner. The reactivity was about 7.2 times at $10 \mathrm{kGy}$ compared with non-irradiated OVA. However, no statistically significant difference was seen for samples between $2 \mathrm{kGy}$ to $6 \mathrm{kGy}$ in electron beam-irradiated OVA, and samples between $4 \mathrm{kGy}$ to $8 \mathrm{kGy}$ in X-ray-irradiated OVA $(p>0.05)$. The binding capacity of P-IgG to electron beamirradiated OVA was slightly higher than that of X-rayirradiated OVA and the pattern of immune reaction was similar to that of M-IgG. However, changes in the doses of 4 to $10 \mathrm{kGy}$-irradiated OVA by X-ray showed differences compared with OVA irradiated by electron beam in the same dose range $(p<0.05)$. Unfortunately, the differences in immune responses of $\mathrm{Ab}$ to $\mathrm{Ag}$ (OVA) irradiated by different sources could not be explained in this study. The differences between radiation sources of immune reaction should be studied further.

OVA can be structurally altered by ionizing radiation, and its antigenicity can be changed. Kang et al. (2002) and Kim et al. (2002) reported the antigenicity (immune response of $\operatorname{IgG}$ ) and allergenicity (immune response of IgE) of ovomucoid and OVA changed by gamma irradiation.

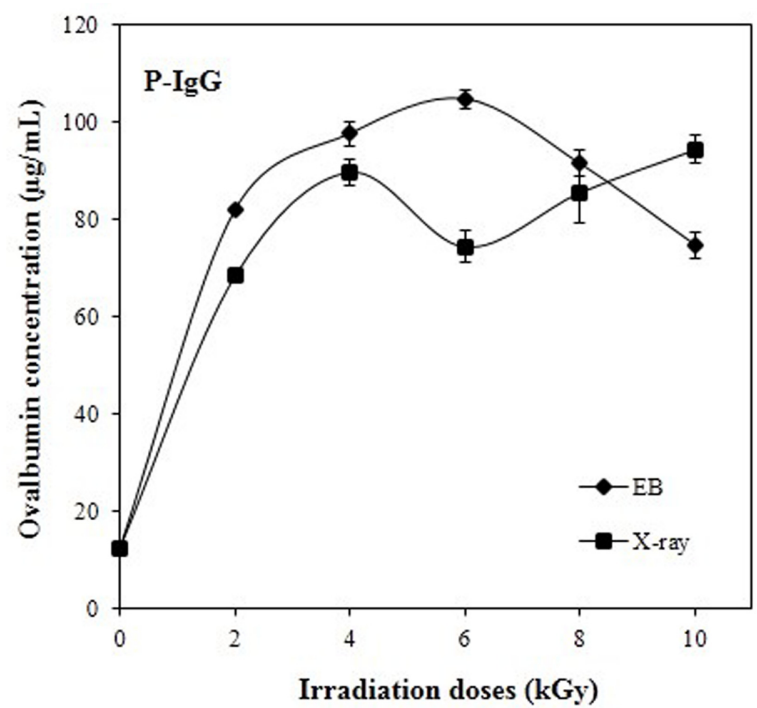

Fig. 3. Mouse polyclonal IgG-binding capacities (detected concentration, $\mu \mathrm{g} / \mathrm{mL}$ ) of each OVA sample irradiated by electron beam or X-ray. The OVA concentrations were individually calculated using equations derived from standard curves obtained by Ci-ELISA formatted with the mouse polyclonal IgG against electron beam-irradiated OVA (Electron beam, - ) or X-ray-irradiated OVA (Xray, $\mathbf{\square})$.

The changes were not dependent upon the irradiation dose and showed a different pattern for antigenicity compared with allergenicity. Seo et al. (2007), and Kume and Matsuda (2005) also reported the changes to conformational structures (epitopes) of gamma-irradiated OVA in their immunological studies. The conformational changes to OVA irradiated by electron beam or X-ray showed different reaction patterns in the mid-range of doses $(<10 \mathrm{kGy})$ in this study. This result indicates differences between radiation sources with respect to changes at the molecular level of a protein.

\section{Conclusions}

Electron beam irradiation and X-ray irradiation showed differences in the structural alterations of OVA by irradiation. Turbidity testing and electrophoretic patterns could not differentiate the differences between radiation sources in irradiated OVA samples. However, in Ci-ELISA, differences in conformational changes were observed between OVA samples irradiated by electron beam or X-ray. The result of these immunological responses indicates that one of the radiation sources should be evaluated and chosen by a preliminary study and a comprehensive molecular study conducted using ionizing radiation prior to 
general acceptance of the application.

\section{Acknowledgements}

This study was supported by the National Research Foundation in the Nuclear Research \& Development Program (2013M2A2A6043298).

\section{References}

1. Al-kahtani, H. A., Abu-Tarbouch, H. M., Atia, M., Bajaber, A. S., Ahmed, M. A., and El-Mojaddidi, M. A. (1998) Amino acid and protein changes in tilapia and Spanish mackerel after irradiation and storage. Radiat. Phys. Chem. 51, 107-114.

2. Byun, M. W., Kim, J. H., Lee, J. W., Park, J. W., Hong, C. S. and Kang, I. J. (2000) Effects of gamma irradiation on the conformational and antigenic properties of a heat-stable major allergen in brown shrimp. J. Food Prot. 63, 940-944.

3. Byun, M. W., Oh, S. H., Kim, J. H., Yoon, Y. H., Park, S. C., Kim, H. S., Kim, S. B., Han, S. B., and Lee, J. W. (2009) Information channel effects on women intention to purchase irradiated food in Korea. Radiat. Phys. Chem. 78, 675-677.

4. Chan, J. K., Gill, T. A., and Paulson, A. T. (1993) Thermal aggregation of myosin subfragments from cod and herring. $J$. Food Sci. 58, 1057-1061.

5. Cho, K. H., Yook, H. S., Lee, J. W., Lee, S. Y., and Byun, M. W. (2001) Changes of binding ability of milk-hypersensitive patients' IgE to gamma-irradiated milk proteins. J. Korean Soc. Food Sci. Nutr. 30, 505-509.

6. Cleland, M. R. and Stichelbaut, F. (2013). Radiation processing with high-energy X-rays. Radiat. Phys. Chem. 84, 91-99.

7. Filali-Mouthim, A., Audette, M., St-louis, M., Thauvett, L., Denoroy, L., Penin, F., Chen, X., Rouleau, N., Le Caer, J. P., Rossier, J., Potier, M., and Le Maire, M. (1997) Lysozyme fragmentation induced by gamma-radiolysis. Int. J. Radiat. Biol. 72, 63-71.

8. Hill, H. D. and Straka, J. G. (1988) Protein determination using bicinchoninic acid in the presence of sulfhydryl reagents. Anal. Biochem. 170, 203-208.

9. Kang, K. O., Lee, J. W., Jo, C. R., Yook H. S., and Byun, M. W. (2002) Changes of allergenicity and conformational structure of egg ovomucoid by gamma irradiation in the basic condition. J. Food Sci. Nut. 7, 52-56.

10. Kim, M. J., Lee, J. W., Yook, H. S., Lee, S. Y., Kim, M. C., and Byun, M. W. (2002) Changes in the antigenic and immunoglobulin E-binding properties of hen's egg albumin with the combination of heat and gamma irradiation treatment. J. Food Prot. 65, 1192-1195.

11. Kume, T., Ishii, T., and Matsuda, T. (1994) Immunochemical identification of irradiated chicken eggs. J. Sci. Food Agric. 65, $1-4$.

12. Kume, T. and Matsuda, T. (1995) Changes in structural and antigenic properties of proteins by radiation. Radiat. Phys. Chem. 46, 225-231.

13. Kuntz, E. and White, E. (1961) Effects of electron beam irradiation on collagen. Federation Pro. 20, 376-391.

14. Laemmli, U. K. (1970). Cleavage of structural proteins during the assembly of the head of bacteriophage T4. Nature 227, 680-685.

15. Lee, J. W., Kim, J. H., Yook, H. S., Kang, K. O., Lee, S. Y., Hwang, H. J., and Byun, M. W. (2000a) Effects of gamma radiation on the allergenic and antigenic properties of milk proteins. J. Food Prot. 64, 272-276.

16. Lee, J. W., Yook, H. S., Lee, K. H., Kim, J. H., Kim, W. J., and Byun, M. W. (2000b) Conformational changes of myosin by gamma irradiation. Radiat. Phys. Chem. 58, 271-277.

17. Lee, J. W., Yook, H. S., Cho, K. H., Lee, S. Y., and Byun, M. W. (2001) The changes of allergenic and antigenic properties of egg white albumin ( Gal d 2) by gamma irradiation. J. Korean Soc. Food Sci. Nutr. 30, 500-504.

18. Lee, J. W. and Byun, M. W. (2003) Food irradiation technology and structural changes of food allergens. J. Asth. Aller. Clin. Immunol. 23, 16-23.

19. Miller, R. B. (2003) Food irradiation using bremsstrahlung X-rays. Radiat. Phys. Chem. 68, 963-974.

20. Palou, L., Marcilla, A., Rojas-Argudo, C., Alonso, M., Jacas, J. A., and del Rio, M. A. (2007) Effects of X-ray irradiation and sodium carbonate treatments on postharvert Penicillium decay and quality attributes of clementine mandarins. Post. Bio. Technol. 46, 252-261.

21. SAS (2008) SAS/STAT Software for PC. Release 9.2, SAS Institute Inc., Cary, NC, USA.

22. Seo, J. H., Kim, J. H., Lee, J. W., Yoo, Y. C., Kim, M. R., Park, K. S., and byun, M. W. (2007) Ovalbumin modified by gamma irradiation alters its immunological functions and allergic responses. Inter. Immunopharm. 7, 464-472.

23. Thayer, D. W. (1994) Wholesomeness of irradiated foods. Food Technol. 48, 58-67.

24. Van Calenberg, S., Van Cleempu, O., Mondelaers, W., and Huyghebaert, A. (1999) Comparison of the effect of X-ray and electron beam irradiation on the microbiological quality of foodstuffs. LWT-Food Sci. Technol. 32, 372-376.

25. Van Calenberg, S., Vanhaelewyn, G., Van Cleemput, O., Callens, F., Mondelaers, W., and Huyghebaert, A. (1998) Comparison of the effect of X-ray and electron beam irradiation on some selected spices. LWT-Food Sci. Technol. 31, 252258.

(Received 2014.4.25/Revised 2014.6.19/Accepted 2014.6.23) 